\title{
Scheduling tasks sharing files on heterogeneous master-slave platforms
}

\author{
Arnaud Giersch ${ }^{\mathrm{a}, *}$, Yves Robert ${ }^{\mathrm{b}}$, Frédéric Vivien ${ }^{\mathrm{b}}$ \\ a ICPS/LSIIT, UMR CNRS-ULP 7005, Parc d'Innovation, Bd Sébastien Brant, BP 10413, \\ 67412 Illkirch Cedex, France \\ ${ }^{\mathrm{b}}$ LIP, UMR CNRS-ENS Lyon-INRIA-UCBL 5668, École normale supérieure de Lyon, \\ 46 allée d'Italie, 69364 Lyon Cedex 07, France
}

Received 30 June 2004; accepted 20 October 2004

Available online 12 July 2005

\begin{abstract}
This paper is devoted to scheduling a large collection of independent tasks onto heterogeneous clusters. The tasks depend upon (input) files which initially reside on a master processor. A given file may well be shared by several tasks. The role of the master is to distribute the files to the processors, so that they can execute the tasks. The objective for the master is to select which file to send to which slave, and in which order, so as to minimize the total execution time. The contribution of this paper is twofold. On the theoretical side, we establish complexity results that assess the difficulty of the problem. On the practical side, we design several new heuristics, which are shown to perform as efficiently as the best heuristics in [H. Casanova, A. Legrand, D. Zagorodnov, F. Berman, Heuristics for scheduling parameter sweep applications in Grid environments, in: Ninth Heterogeneous Computing Workshop, IEEE Computer Society Press, Silver Spring, MD, 2000, pp. 349-363; H. Casanova, A. Legrand, D. Zagorodnov, F. Berman, Using simulation to evaluate scheduling heuristics for a class of applications in Grid environments, Research Report RR-1999-46, LIP, ENS Lyon, France, 1999] although their cost is an order of magnitude lower.
\end{abstract}

(c) 2005 Elsevier B.V. All rights reserved.

Keywords: Scheduling; Heterogeneous clusters; Independent tasks; File-sharing; Heuristics

\section{Introduction}

${ }^{*}$ Corresponding author. Tel.: +33 3902445 42; fax: +333 90244547.

E-mail addresses: arnaud.giersch@icps.u-strasbg.fr (A. Giersch),yves.robert@ens-lyon.fr (Y. Robert), frederic.vivien@ ens-lyon.fr (F. Vivien).
In this paper, we are interested in scheduling independent tasks onto heterogeneous clusters. These independent tasks depend upon files (corresponding to input data, for example), and 
difficulty arises from the fact that some files may well be shared by several tasks.

This paper is motivated by the work of Casanova et al. [1,2], who target the scheduling of tasks in APST, the AppLeS Parameter Sweep Template [3]. APST is a grid-based environment whose aim is to facilitate the mapping of applications to heterogeneous platforms. Typically, an APST application consists of a large number of independent tasks, with possible input data sharing (see $[1,2]$ for a detailed description of a real-world application). By large we mean that the number of tasks is usually at least one order of magnitude larger than the number of available computing resources. When deploying an APST application, the intuitive idea is to map those tasks that depend upon the same files onto the same computational resource, so as to minimize communication requirements. Casanova et al. $[1,2]$ have considered three heuristics designed for completely independent tasks (no input file sharing) that were proposed in [4]. They have modified these three heuristics (originally called Min-min, Max-min, and Sufferage in [4]) to adapt them to the additional constraint that input files are shared between tasks. As was already pointed out, the number of tasks to schedule is expected to be very large, and special attention should be devoted to keeping the cost of the scheduling heuristics reasonably low.

In this paper, we deal with the same scheduling problem as Casanova et al. [1,2]: we assume the existence of a master processor, which serves as the repository for all files. The role of the master is to distribute the files to the processors, so that they can execute the tasks. The objective for the master is to select which file to send to which slave, and in which order, so as to minimize the total execution time. This master-slave paradigm has a fundamental limitation: communications from the master may well become the true bottleneck of the overall scheduling scheme. Allowing for inter-slave communications, and/or for distributed file repositories, should certainly be the subject of future work. However, we believe that concentrating on the simpler master-slave paradigm is a first but mandatory step towards a full understanding of this challenging scheduling problem.
The contribution of this paper is twofold. On the theoretical side, we establish two complexity results that assess the difficulty of the problem:

- The first result shows the NP-completeness of the scheduling problem with a single slave.

- The second result shows the NP-completeness of the scheduling problem with two slaves, in the special case where all tasks and files have same size.

On the practical side, we design several new heuristics, which are shown to perform as efficiently as the best heuristics in $[1,2]$ although their cost is an order of magnitude lower.

The rest of the paper is organized as follows. The next section (Section 2) is devoted to the precise and formal specification of our scheduling problem, which we denote as TASKSSHARINGFiLEs. Next, in Section 3, we state complexity results, which include the two NP-completeness results already mentioned. Then, Section 4 deals with the design of low-cost polynomial-time heuristics to solve the TASKsSharingFiLes problem. We report some experimental data in Section 5. Finally, we state some concluding remarks in Section 6.

\section{Framework}

In this section, we formally state the optimization problem to be solved.

\subsection{Tasks and files}

The problem is to schedule a set of $n$ tasks $\mathscr{T}=\left\{T_{1}, T_{2}, \ldots, T_{n}\right\}$. These tasks have different sizes: the weight of task $T_{j}$ is $t_{j}, 1 \leqslant j \leqslant n$. There are no dependence constraints between the tasks, so they can be viewed as independent.

However, the execution of each task depends upon one or several files, and a given file may be shared by several tasks. Altogether, there are $m$ files in the set $\mathscr{F}=\left\{F_{1}, F_{2}, \ldots, F_{m}\right\}$. The size of file $F_{i}$ is $f_{i}, 1 \leqslant i \leqslant m$. We use a bipartite graph $\mathscr{G}=(\mathscr{V}, \mathscr{E})$ to represent the relations between files and tasks. The set of nodes in the graph $\mathscr{G}$ is $\mathscr{V}=\mathscr{F} \cup \mathscr{T}$, and each node is weighted by $f_{i}$ or $t_{j}$, depending 


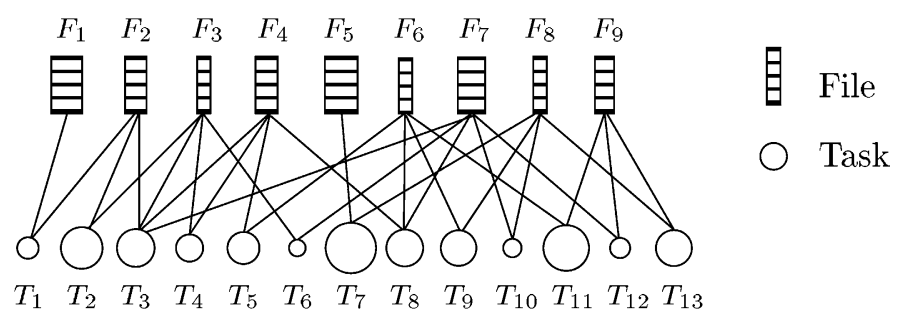

Fig. 1. Bipartite graph gathering relations between files and tasks.

upon its membership in $\mathscr{F}$ or $\mathscr{T}$. There is an edge $e_{i, j}: F_{i} \rightarrow T_{j}$ in $\mathscr{E}$ if and only if task $T_{j}$ depends on file $F_{i}$. Intuitively, files $F_{i}$ such that $e_{i, j} \in \mathscr{E}$ correspond to some data that is needed for the execution of $T_{j}$ to begin. The processor that will have to execute task $T_{j}$ will need to receive all the files $F_{i}$ such that $e_{i, j} \in \mathscr{E}$ before it can start the execution of $T_{j}$. See Fig. 1 for a small example, with $m=9$ files and $n=13$ tasks. For instance, task $T_{1}$ depends upon files $F_{1}$ and $F_{2}$, and task $T_{3}$ depends upon files $F_{2}, F_{3}, F_{4}$, and $F_{7}$.

To summarize, the bipartite application graph $\mathscr{G}=(\mathscr{V}, \mathscr{E})$, where each node in $\mathscr{V}=\mathscr{F} \cup \mathscr{T}$ is weighted by $f_{i}$ or $t_{j}$, and where edges in $\mathscr{E}$ represent the relations between the files and the tasks, gathers all the information on the application.

\subsection{Platform graph}

The tasks are scheduled and executed on a master-slave heterogeneous platform. We let $\mathscr{P}$ denote the platform graph, which is a fork-graph (see Fig. 2) with a master-processor $P_{0}$ and $p$ slaves $P_{i}$, $1 \leqslant i \leqslant p$. Each slave $P_{q}$ has a (relative) cycle time $w_{q}$ : it takes $t_{j} \cdot w_{q}$ time-units to execute task $T_{j}$ on processor $P_{q}$. We point out that all the results and heuristics of this paper can straightforwardly

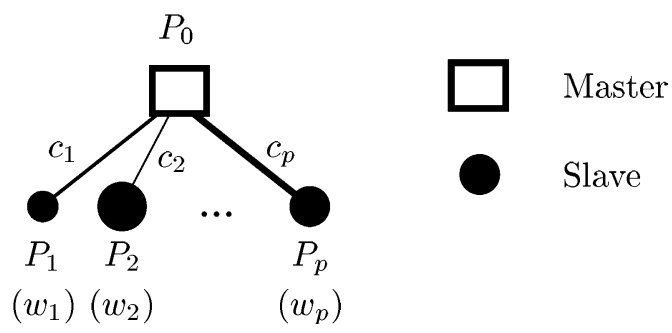

Fig. 2. Heterogeneous fork-graph. be extended to the more general case of inconsistent execution times, with the terminology of [5]: in that situation, each slave $P_{q}$ has a different execution time $w_{j, q}$ for each task $T_{j}$, and these times are not related; then, we would simply replace all terms $t_{j} \cdot w_{q}$ by $w_{j, q}$.

The master processor $P_{0}$ initially holds all the $m$ files in $\mathscr{F}$. The slaves are responsible for executing the $n$ tasks in $\mathscr{T}$. Before it can execute a task $T_{j}$, a slave must have received from the master all the files that $T_{j}$ depends upon. For communications, we use the one-port model: the master can only communicate with a single slave at a given timestep. We let $c_{q}$ denote the inverse of the bandwidth of the link between $P_{0}$ and $P_{q}$, so that $f_{i} \cdot c_{q}$ timeunits are required to send file $F_{i}$ from the master to the slave $P_{q}$. We assume that communications can overlap computations on the slaves: a slave can process one task while receiving the files necessary for the execution of another task.

Coming back to the example of Fig. 1, assume that we have a two-slave schedule such that tasks $T_{1}$ to $T_{6}$ are executed by slave $P_{1}$, and tasks $T_{7}$ to $T_{13}$ are executed by slave $P_{2}$. Overall, $P_{1}$ will receive six files $\left(F_{1}\right.$ to $F_{4}, F_{6}$, and $\left.F_{7}\right)$, and $P_{2}$ will receive six files $\left(F_{4}\right.$ to $\left.F_{9}\right)$. In this schedule, three files $\left(F_{4}, F_{6}\right.$, and $\left.F_{7}\right)$ must be sent to both slaves.

To summarize, we assume a fully heterogeneous master-slave paradigm: slaves have different speeds and links have different capacities. Communications from the master are serial, and may well become the major bottleneck.

\subsection{Objective function}

The objective is to minimize the total execution time. The execution is terminated when the last task has been completed. The schedule must decide 
which tasks will be executed by each slave. It must also decide the ordering in which the master sends the files to the slaves. We stress two important points:

- Some files may well be sent several times, so that several slaves can independently process tasks that depend upon these files.

- A file sent to some processor remains available for the rest of the schedule. If two tasks depending on the same file are scheduled on the same processor, the file must only be sent once to that processor.

To decrease the total execution time, we may will try to limit the amount of replicated files. By mapping on a same processor tasks depending on a same file, the communication time will be reduced. But then there is the risk that all tasks are mapped on a single processor. On the contrary, if we try to balance the load between the processors, a lot of communications may be induced. There is a trade-off to be found between these two extreme solutions.

We let TAsksSharingFiles $(\mathscr{G}, \mathscr{P})$ denote the optimization problem to be solved.

\section{Complexity}

Most scheduling problems are known to be difficult $[6,7]$. However, some particular instances of the TAsksSharingFiles optimization problem have a polynomial complexity, while the decision problems associated to other instances are NPcomplete. We outline several results in this section, which are all gathered in Fig. 3. In Fig. 3, the pictographs read as follows: for each of the six case studies, the leftmost diagram represents the application graph, and the rightmost diagram represents the platform graph. We draw objects of different sizes to symbolically represent their heterogeneity. The application graph is made up of files and tasks which all have the same sizes in situations (a)-(c), while this is not the case in situations (d)-(f). Tasks depend upon a single (private) file in situations (a), (b), (d) and (e), which is not the case in situations (c) and (f). As for the platform graph, there is a single slave in situations (d) and (f), and several slaves otherwise. The platform is homogeneous in cases (a) and (e), and heterogeneous in cases (b) and (c). The six situations are discussed in the text below.

\subsection{With a single slave}

The instance of TASKsSHARINGFiLes with a single slave turns out to be more difficult than we would think intuitively. In the very special case where each task depends upon a single non-shared file, i.e., $n=m$ and $\mathscr{E}$ reduces to $n$ edges $e_{i, i}$ : $F_{i} \rightarrow T_{i}$, the problem can be solved in polynomial time (this is situation (d) of Fig. 3). Indeed, it is equivalent to the two-machine flow-shop problem,

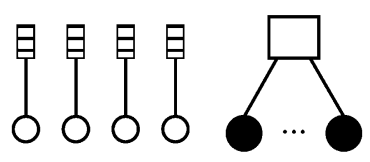

a

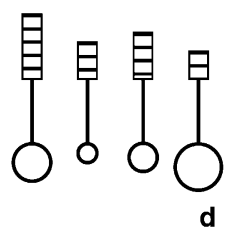

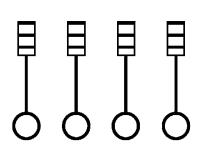

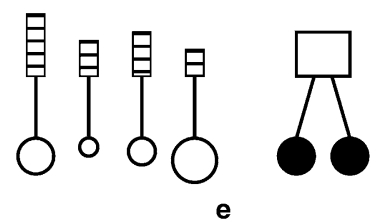

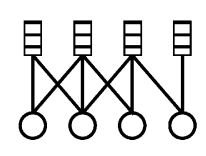

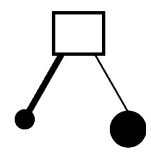

c

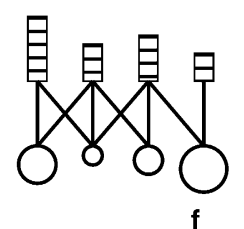

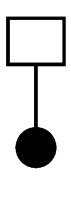

Fig. 3. Complexity results for the problem of scheduling tasks sharing files: (a) polynomial (round-robin); (b) polynomial [8]; (c) NPcomplete (this paper); (d) polynomial [9] (e) NP-complete (2-PARTITION) (f) NP-complete (this paper). 
and the algorithm of Johnson [9] can be used to compute the optimal execution time. According to Johnson's algorithm we first schedule the tasks whose communication time (the time needed to send the file) is smaller than (or equal to) the execution time in increasing order of the communication time. Then we schedule the remaining tasks in decreasing order of their execution time.

The general instance with a single slave, where files are shared between tasks, corresponds to situation (f) of Fig. 3. One major result of this paper is to prove the NP-hardness of this instance. Interestingly, this shows that (unless $\mathrm{P}=\mathrm{NP}$ ) there is no polynomial algorithm to extend Johnson's algorithm for general graphs.

The decision problem associated to the general instance of TASKSSHARINGFILES with a single slave can formally be stated as follows:

Definition 1 (TSF1-DeC $(\mathscr{G}, \mathscr{P}, p=1, K)$ ). Given a bipartite application graph $\mathscr{G}$, a platform $\mathscr{P}$ with a single slave $(p=1)$ and a time bound $K$, is it possible to schedule all tasks within $K$ timesteps?

Theorem 1. TSF1-DeC $(\mathscr{G}, \mathscr{P}, p=1, K) \quad$ is $\quad N P$ complete.

Proof. Obviously, $\operatorname{TSF} 1-\operatorname{Dec}(\mathscr{G}, \mathscr{P}, p=1, K)$ belongs to NP. To prove its completeness, we use a reduction from MEWC, the Maximum EdgeWeighted Clique problem, which is NP-complete [10]. Consider an arbitrary instance $\mathscr{I}_{1}$ of MEWC: given a complete edge-weighted graph $G_{c}=\left(V_{c}\right.$, $\left.E_{c}, w\right)$, where $w: \quad E_{c} \rightarrow \mathbb{N}$ is the weight function, a size bound $B$, where $3 \leqslant B \leqslant\left|V_{c}\right|$, and a weight bound $W>0$, is there a subset $S$ of $B$ vertices such that $\sum_{e \in E_{S}} w(e) \geqslant W$ ? Here, $E_{S}$ denotes the set of the $B \cdot(B-1) / 2$ edges connecting the vertices of $S$. In other words, can we find $B$ vertices inducing a sub-graph of weight at least $W$ ? We point out that the original formulation of MEWC in [10] asks for a subset of at most $B$ vertices rather than of exactly $B$ vertices, as we do here. However, it is straightforward to see that our formulation remains NP-complete (any polynomial algorithm solving our formulation could be invoked at most $|V|$ times to solve the original formulation).

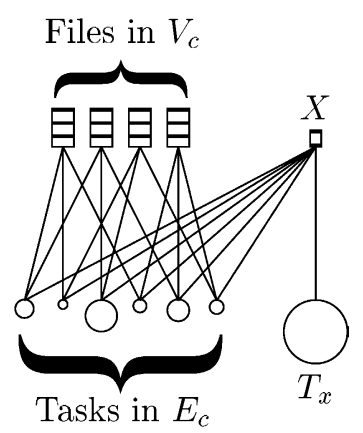

Fig. 4. The bipartite application graph used in the proof of Theorem 1, with $\left|V_{c}\right|=4$.

We construct the following instance $\mathscr{I}_{2}$ of $\operatorname{TSF} 1-\operatorname{DeC}(\mathscr{G}, \mathscr{P}, p=1, K)$. We let $\mathscr{F}=V_{c} \cup\{X\}$ and $\mathscr{T}=E_{c} \cup\left\{T_{x}\right\}$ (see Fig. 4), which defines $\mathscr{V}=\mathscr{F} \cup \mathscr{T}$. There are $m=\left|V_{c}\right|+1$ files, and $n=\left|E_{c}\right|+1=(m-1) \cdot(m-2) / 2+1$ tasks (the original graph $G_{c}$ is complete, hence $\left|E_{c}\right|=\left|V_{c}\right|$. $\left.\left(\left|V_{c}\right|-1\right) / 2\right)$.

The size of file $X$ is 1 , and the size of each file corresponding to a node in $V_{c}$ is $f=W \cdot(2 B-1)$. The weight of task $T_{x}$ is $x=W \cdot\left(B^{2}+2 B-2\right)$. Note that $x \geqslant 0$ because $B \geqslant 3$. The weight of the task corresponding to an edge $e \in E_{c}$ is $2 W+w(e)$.

The relations between tasks and files are defined as follows. First, there is an edge from file $X$ to each task in $\mathscr{T}$. Second, there is an edge from a node (file) $v \in V_{c} \subset \mathscr{F}$ to a node (task) $e \in E_{c} \subset \mathscr{T}$ if and only if $v$ was one of the two end-points of edge $e$ in $G_{c}$. As a consequence, each edge-task (in $\mathscr{T} \backslash\left\{T_{x}\right\}$ ) exactly depends upon three files ( $X$, and both end-points of the edge). The computing platform is quite simple: a single slave, with unit communication and computation time: $c_{1}=w_{1}=1$. Finally, we define the scheduling bound:

$K=1+x+\sum_{e \in E_{c}} w(e)+2 W \cdot\left|E_{c}\right|$

Clearly, the instance $\mathscr{I}_{2}$ can be constructed in time polynomial in the size of $\mathscr{I}_{1}$. Now we have to show that $\mathscr{I}_{2}$ admits a solution if and only if $\mathscr{I}_{1}$ has one.

Assume first that $\mathscr{I}_{1}$ has a solution, i.e., that $G_{c}$ possesses $B$ vertices inducing a sub-graph whose edge-weight is at least $W$. Let $\mathscr{C}=\left\{v_{1}, v_{2}, \ldots, v_{B}\right\}$ 
denote these $B$ vertices. The intuitive idea to construct the schedule is as follows: after sending file $X$, the master sends the $B$ files corresponding to the $B$ nodes in $\mathscr{C}$. Because these files induce a large amount of work, the slave processor will have enough work to process while receiving the other files. The idea is to keep the slave processor active all the time as soon as it has received file $X$. The bound $K$ is defined accordingly: the first time-unit is spent receiving $X$, and the rest amounts to the sum of all task weights.

The schedule is defined as follows:

(1) At time-step $t=0$, file $X$ is sent to the slave.

(2) The master sends the files (corresponding to the nodes) of $V_{c}$ as soon as possible, i.e., at time $t=1+(j-1) \cdot f$ for the $j$ th file, $1 \leqslant j \leqslant\left|V_{c}\right|$ (recall that $f$ is the size of each file in $V_{c}$ ). The first $B$ files sent are chosen to be those in $\mathscr{C}$, in any order. The remaining $\left|V_{c}\right|-B$ files are then sent in any order.

(3) The slave has an execution queue, which it processes greedily, and in FIFO order. At time-step $t=1, T_{x}$ is available in the queue, and the slave starts its execution. Upon reception of the first file of $V_{c}$, no new task is ready. But upon reception of the $j$ th file of $V_{c}$, with $j \geqslant 2$, there are $j-1$ new tasks ready for execution: they correspond to all the edges in $G_{c}$ whose first end-point is the $j$ th file, and whose other end-point is one of the $j-1$ files of $V_{c}$ previously received. These $j-1$ tasks are inserted at the end of the execution queue, in any order.

We have derived a schedule for instance $\mathscr{I}_{2}$, but does it match the execution bound $K$ ? As already mentioned, this is only possible if the slave is never idle after receiving file $X$. Let $\operatorname{RC}(j)$ denotes the receive capacity of the slave upon reception of the $j$ th file from $V_{c}: \operatorname{RC}(j)$ denotes the amount of work that remains to be executed for the current task and those ready in the queue. It corresponds to the time the processor can spend, waiting for a new file, without becoming inactive. Similarly, $\mathrm{RC}(0)$ denotes the receive capacity of the slave upon reception of file $X$ i.e., the time to execute task $T_{x}$.
Obviously, we would like $\operatorname{RC}(j) \geqslant f$ for all $j \geqslant 0$ : this would allow the slave to receive a new file without becoming idle.

Initially, owing to task $T_{x}$, we have $\mathrm{RC}(0)=x$. Let $E_{j}$ denote the set of the tasks from $E_{c}$ that only depend on the first $j$ files from $V_{c}$ sent to the slave. We have $\mathrm{RC}(1)=x-f$ (the first file does not grant any work), and

$\mathrm{RC}(j)=x+\sum_{e \in E_{j}} w(e)+2 W \cdot\left|E_{j}\right|-f \cdot j$

for all $j \geqslant 2$. Indeed, this quantity is the sum of the execution times of the tasks in $E_{j} \cup\left\{T_{x}\right\}$, minus the time spent to send the first $j$ files. We want to show that $\mathrm{RC}(j) \geqslant f$ for all $j, 0 \leqslant j \leqslant\left|V_{c}\right|$. We have $\mathrm{RC}(0)-f=x-f \geqslant x-2 f=\mathrm{RC}(1)-f$. Furthermore, $x-2 f=W \cdot\left(B^{2}-2 B\right) \geqslant 0$, since $B \geqslant 3$. So $\mathrm{RC}(0) \geqslant f$ and $\mathrm{RC}(1) \geqslant f$. For $j \geqslant 2,\left|E_{j}\right|=j$. $(j-1) / 2$, and $\mathrm{RC}(j)-f=\sum_{e \in E_{j}} w(e)-W+h(j)$, where $h(j)=W \cdot(j-B)^{2}$. The minimum of $h(j)$ is zero, and is obtained for $j=B$. But due to the choice of the first $B$ files sent to the slave, $\sum_{e \in E_{B}} w(e) \geqslant W$, hence $\operatorname{RC}(B)-f \geqslant 0$. For $j \neq B$, $h(j) \geqslant h(B-1)=h(B+1)=W$ and $\operatorname{RC}(j)-f \geqslant$ $h(j)-W \geqslant 0$. Altogether, this concludes the proof that the total execution of the schedule is equal to $K$, hence a solution to $\mathscr{I}_{2}$.

Assume now that $\mathscr{I}_{2}$ has a solution. We have a schedule with executes within $K=1+x+$ $\sum_{e \in E_{c}} w(e)+2 W \cdot\left|E_{c}\right|$ time-units. But $K$ is equal to one plus the sum of the task weights. Because the slave processor is idle until file $X$ has been sent, necessarily the first file sent is $X$, and this emission lasts one time-unit. After the first time-step, the slave processor must be kept busy all the time. Letting $E_{j}$ be the set of the tasks from $E_{c}$ that only depend of the first $j$ files of $V_{c}$ sent to the slave, we must have as previously, $\mathrm{RC}(j) \geqslant f$ for all $1 \leqslant$ $j \leqslant\left|V_{c}\right|$. For $j \geqslant 2$, we know that $\operatorname{RC}(j) \leqslant x+$ $\sum_{e \in E_{j}} w(e)+2 W \cdot\left|E_{j}\right|-f \cdot j$. We had an equality before, but maybe the schedule did not send the files as soon as possible, hence the inequality here. Taking $j=B$ we derive just as before that $\mathrm{RC}(B)-f \leqslant \sum_{e \in E_{B}} w(e)-W$. Because the slave is never idle after receiving the $B$-th file, we have $\mathrm{RC}(B)-f \geqslant 0$, and we derive that $\sum_{e \in E_{B}} w(e) \geqslant$ $W$. The first $B$ files sent to the slave provide a solution to $\mathscr{I}_{1}$. 


\subsection{With two slaves}

With several slaves, some problem instances have polynomial complexity. First of all, a greedy round-robin algorithm is optimal in situation (a) of Fig. 3: each task depends upon a single nonshared file, all tasks and files have the same size, and the fork platform is homogeneous. If we keep the same hypotheses for the application graph but move to heterogeneous slaves (situation (b) of Fig. 3 ), the problem remains polynomial, but the optimal algorithm becomes complicated: see [8] for a description and proof.

The decision problem associated to the general instance of TASKSSHARINGFILES with two slaves, writes as follows:

Definition 2 (TSF2-DEC $(\mathscr{G}, \mathscr{P}, p=2, K)$ ). Given a bipartite application graph $\mathscr{G}$, a heterogeneous platform $\mathscr{P}$ with two slaves $(p=2)$, and a time bound $K$, is it possible to schedule all tasks within $K$ time-steps?

Clearly, TSF2-Dec is NP-complete, even if there are no files at all: in that case, TSF2-DEC reduces to the scheduling of independent tasks on a two-processor machine, which itself reduces to the 2-PARTITION problem [11] as the tasks have different sizes. This corresponds to situation (e) in Fig. 3, where we do not even need the private files. However, this NP-completeness result does not hold in the strong sense: in a word, the size of the tasks plays a key role in the proof, and there are pseudo-polynomial algorithms to solve TSF2-DEC in the simple case when there are no files (see the pseudo-polynomial algorithm for 2-PARTITION in [11]).
The following theorem states an interesting result: in the case where all files and tasks have unit size (i.e., $f_{i}=t_{j}=1$ ), the TSF2-DEC remains NPcomplete. Note that in that case, the heterogeneity only comes from the computing platform. This corresponds to situation (c) in Fig. 3.

Definition 3 (TSF2-EQuAL-DeC $\left(\mathscr{G}, \mathscr{P}, p=2, f_{i}=\right.$ $\left.t_{j}=1, K\right)$ ). Given a bipartite application graph $\mathscr{G}$ such that $f_{i}=t_{j}=1$ for all tasks and files, a heterogeneous platform $\mathscr{P}$ with two slaves $(p=2)$, and a time bound $K$, is it possible to schedule all tasks within $K$ time-steps?

Theorem 2. TSF2-EQUAL-DeC $\left(\mathscr{G}, \mathscr{P}, p=2, f_{i}=t_{j}=\right.$ $1, K)$ is NP-complete.

Proof. Obviously, TSF2-EQUAL-Dec $(\mathscr{G}, \mathscr{P}, p=2$, $\left.f_{i}=t_{j}=1, K\right)$ belongs to NP. To prove its completeness, we use a reduction from Clique, which is NP-complete [11]. Consider an arbitrary instance $\mathscr{I}_{1}$ Of CliQUE: given a graph $G_{c}=\left(V_{c}, E_{c}\right)$, and a bound $B$, is there a clique in $G_{c}$ (i.e., a fully connected sub-graph) of size $B$ ? Without loss of generality, we assume that $\left|V_{c}\right| \geqslant 9$ and $6 \leqslant B$. $(B-1)<\left|E_{c}\right|$.

We construct the following instance $\mathscr{I}_{2}$ of TSF2-EQUAL-DeC $\left(\mathscr{G}, \mathscr{P}, p=2, f_{i}=t_{j}=1, K\right)$. We let $\mathscr{F}=V_{c} \cup X$ and $\mathscr{T}=E_{c} \cup\left\{T_{y}\right\}$ (see Fig. 5), which defines $\mathscr{V}=\mathscr{F} \cup \mathscr{T}$. Here, $X$ is a collection of $x=|X|$ additional files, so there is a total of $\left|V_{c}\right|+x$ files, one per node in the original graph $G_{c}$ and one per new file in $X$. As for tasks, there are as many tasks as edges in the original graph $G_{c}$, plus an additional task $T_{y}$.

The relations between tasks and files are defined as follows. First, there is an edge from each file in

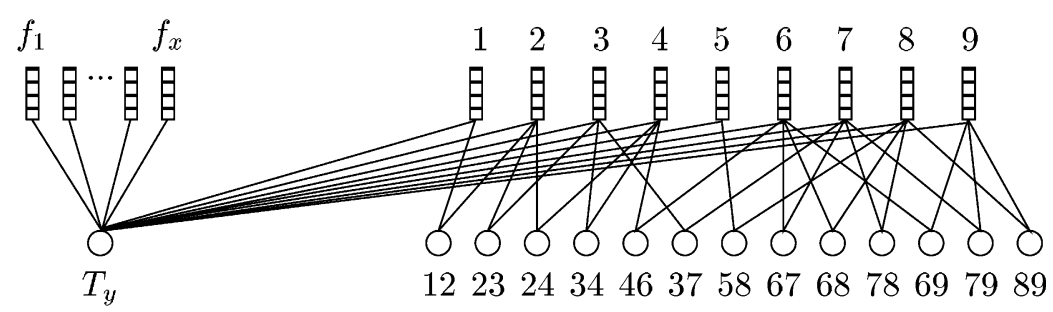

Fig. 5. The bipartite application graph used in the proof of Theorem 2. 


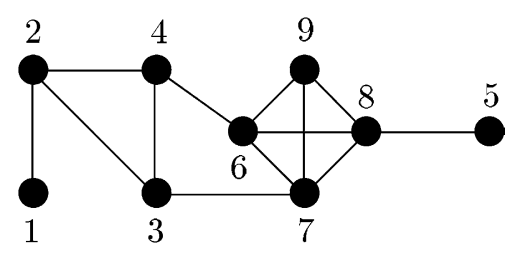

Fig. 6. The original graph $G_{c}$ used to build the bipartite graph of Fig. 5.

$\mathscr{F}$ to task $T_{y}$; as a consequence, the slave processor that will execute $T_{y}$ will need to have received all the files in $\mathscr{F}$ from the master before it can begin the execution of $T_{y}$. Second, there is an edge from a node (file) $v \in V_{c} \subset \mathscr{F}$ to a node (task) $e \in E_{c} \subset \mathscr{T}$ if and only if $v$ was one of the two end-points of edge $e$ in $G_{c}$. In the rightmost part of Fig. 5, the bipartite graph has been obtained from the original graph $G_{c}$ shown in Fig. 6. The files are the nodes in $G_{c}$, and the tasks are the edges in $G_{c}$. This explains why each task (edge) exactly depends upon two files (the end-points of the edge). We see that $G_{c}$ has a clique of size $B=4$ (nodes 6-9).

As specified in the problem, all files and tasks have unit size. To complete the description of the application, we let $s=B \cdot(B-1) / 2, r=\left|E_{c}\right|-s$ (note that $s<r$ by hypothesis), and we define $x=(3 r-1) \cdot\left|V_{c}\right|-2 B+2$. We check that $x \geqslant 1$ : indeed, $r \geqslant 4$ and $\left|V_{c}\right| \geqslant B$; we derive $x \geqslant 9 B+2$.

There remains to describe the computing platform. The characteristics of the two slave processors are: $w_{1}=3 \cdot\left|V_{c}\right|, w_{2}=\left(3 \cdot(r+1) \cdot\left|V_{c}\right|-4\right) / s$, $c_{1}=1$, and $c_{2}=2$. Note that $w_{2}>w_{1}$, because $w_{2}-w_{1}=3 \cdot(r / s-1) \cdot\left|V_{c}\right|+\left(3 \cdot\left|V_{c}\right|-4\right) / s>0$. Thus, $w_{2}>2$. Finally, we define the scheduling bound:

$K=2+3 \cdot(r+1) \cdot\left|V_{c}\right|=6+s \cdot w_{2}$.

Clearly, the instance $\mathscr{I}_{2}$ can be constructed in time polynomial in the size of $\mathscr{I}_{1}$. Now we have to show that $\mathscr{I}_{2}$ admits a solution if and only if $\mathscr{I}_{1}$ has one.

Assume first that $\mathscr{I}_{1}$ has a solution, i.e., that $G_{c}$ possesses a clique of size $B$. Let $\mathscr{C}=\left\{v_{1}, v_{2}, \ldots, v_{B}\right\}$ denote the $B$ vertices in the clique of $G_{c}$. The intuitive idea is the following: after sending to slave $P_{2}$ the $B$ files corresponding to the $B$ nodes in $\mathscr{C}, P_{2}$ will be able to process the $s$ tasks that correspond to the edges connecting the nodes of $\mathscr{C}$ without receiving any extra file from the master. The schedule is defined as follows:

- First, at time-steps $t=0$ and $t=1$, two files are sent by the master to $P_{1}$. These two files are chosen so that they correspond to any two nodes $v_{a}$ and $v_{b}$ of $V_{c}$ that are connected in $G_{c}$ (i.e., the edge $\left(v_{a}, v_{b}\right)$ belongs to $\left.E_{c}\right)$ and that do not both belong to the clique $\mathscr{C}$. Note that such an edge exists, as the number of edges with their two end-points in $\mathscr{C}$ if $B \cdot(B-1) /$ $2<\left|E_{c}\right|$ (by hypothesis). At time-step $t=2$, $P_{1}$ is able to start the execution of the task that corresponds to the edge $\left(v_{a}, v_{b}\right) . P_{1}$ terminates this task at time-step $2+w_{1}=$ $2+3 \cdot\left|V_{c}\right|$.

- Next, the $B$ files that correspond to the clique $\mathscr{C}$ are sent to $P_{2}$. As soon as it has received two files, $P_{2}$ can start executing one task (the two files correspond to two connected nodes, therefore the task that represents the edge between them is ready for execution). $P_{2}$ has received the $B$ files at time-step $2 c_{1}+B \cdot c_{2}=2+2 B$, i.e., before it completes the execution of the first task, at time-step $2 c_{1}+2 c_{2}+w_{2}=6+w_{2}>6+$ $w_{1}=6+3 \cdot\left|V_{c}\right| \geqslant 6+3 B$, because $B \leqslant\left|V_{c}\right|$. Therefore, $P_{2}$ can process the $s$ tasks corresponding to edges in the clique $\mathscr{C}$ without interruption (i.e., without waiting to receive more files), until time-step $2 c_{1}+2 c_{2}+s \cdot w_{2}=6+$ $s \cdot w_{2}=K$.

- Finally, after sending the $B$ files to $P_{2}$, all files but two are sent to $P_{1}$ : we do not re-send the first two files, but we send all the others, i.e., $\left|V_{c}\right|-2+x$ files. We send the $\left|V_{c}\right|-2$ files corresponding to nodes in $V_{c}$ before the $x$ files corresponding to nodes in $X$. When $P_{1}$ terminates its first task, at time-step $2+3 \cdot\left|V_{c}\right|$, it has already received the first $\left|V_{c}\right|-2$ files (the last one is received at time-step $2 c_{1}+B \cdot c_{2}+$ $\left.\left(\left|V_{c}\right|-2\right) \cdot c_{1}=\left|V_{c}\right|+2 B\right) . P_{1}$ can process the $r$ tasks corresponding to edges in $G_{c}$ that do not belong to the clique $\mathscr{C}$ without interruption, until time-step $2 c_{1}+r \cdot w_{1}=K-w_{1}$. At that time-step, $P_{1}$ has just received the $x$ last files, 
because $\left(\left|V_{c}\right|+x\right) \cdot c_{1}+B \cdot c_{2}=K-w_{1} . P_{1}$ processes then the last task $T_{y}$, and the scheduling terminates within $K$ times-steps.

We have derived a valid solution to our scheduling instance $\mathscr{I}_{2}$.

Assume now that $\mathscr{I}_{2}$ has a solution. We proceed in several steps:

(1) Necessarily, $P_{1}$ executes task $T_{y}$. Otherwise, $P_{2}$ would execute it, but $T_{y}$ requires $\left|V_{c}\right|+x$ files, and the time needed by $P_{2}$ would be at least $\left(\left|V_{c}\right|+x\right) \cdot c_{2}+w_{2}=2 \cdot\left(K-w_{1}-2 B\right)+$ $w_{2}>2 \cdot\left(K-5 \cdot\left|V_{c}\right|\right)>K$ (because $K \geqslant 15$. $\left.\left|V_{c}\right|\right)$, a contradiction.

(2) $P_{1}$ cannot execute more than $\left(K-2 c_{1}\right) /$ $w_{1}=r+1$ tasks, because it must have received two files before it can start to process its first task.

(3) All files sent by the master after time-step $K-w_{1}$ are useless, because the tasks that they might free for execution will not be terminated at time-step $K$, neither by $P_{1}$ nor by $P_{2}$ (remember that $w_{2}>w_{1}$ ). Because $P_{1}$ executes $T_{y}$, it receives $\left|V_{c}\right|+x$ files. But $K-w_{1}=\left(\left|V_{c}\right|+x\right) \cdot c_{1}+B \cdot c_{2}$, so that $P_{2}$ cannot receive more than $B$ tasks from the master.

(4) $P_{2}$ cannot execute more than $s$ tasks, because $\left(K-2 c_{2}\right) / w_{2}=(K-6) / w_{2}+2 / w_{2}=$ $s+2 / w_{2}<s+1$.

Overall, a total of $r+s+1$ tasks are executed. Since $P_{1}$ cannot execute more than $r+1$ tasks, and $P_{2}$ more than $s$ tasks, they do execute $r+1$ and $s$ tasks respectively. But $P_{2}$ executes $s$ tasks and receives no more than $B$ files: these files define a clique of size $B$ in $G_{c}$, thereby providing a solution to $\mathscr{I}_{1}$.

Finally, we have shown that the decision problem associated with TASKsSHARINGFILES is NPcomplete, even in the simple cases where:

(1) there is a single slave, but tasks and files have heterogeneous sizes;

(2) tasks and files have unitary size, but the platform is composed of two heterogeneous pro- cessors connected with links of different bandwidths.

At the time of this writing, we do not know the complexity of the problem instance where the platform is homogeneous and tasks and files have unitary size. We do not even know the complexity when there is a single slave (and homogeneous tasks and sizes).

In the general version of the problem, everything is heterogeneous: the sizes of the tasks and of the files are different, the slave processors have different speeds and are connected to the master with links of different bandwidths. Therefore, in the following, we design polynomial heuristics to solve the TASKsSharingFiles problem, and we assess their performance trough extensive simulations.

\section{Heuristics}

In this section, we first recall the three heuristics used by Casanova et al. [1,2]. Next we introduce several new heuristics, whose main characteristic is a lower computational complexity.

\subsection{Reference heuristics}

Because our work was originally motivated by the paper of Casanova et al. [1,2], we have to compare our new heuristics to those presented by these authors, which we call reference heuristics. We start with a description of these reference heuristics.

\subsubsection{Structure of the heuristics}

All the reference heuristics are built on the model presented by Algorithm 1: while there remain tasks to be scheduled, an objective function is evaluated for all pairs of a task (which remains to be scheduled) and a processor. The task that will actually be scheduled, as well as the target processor, are chosen according to the values of this objective function. 


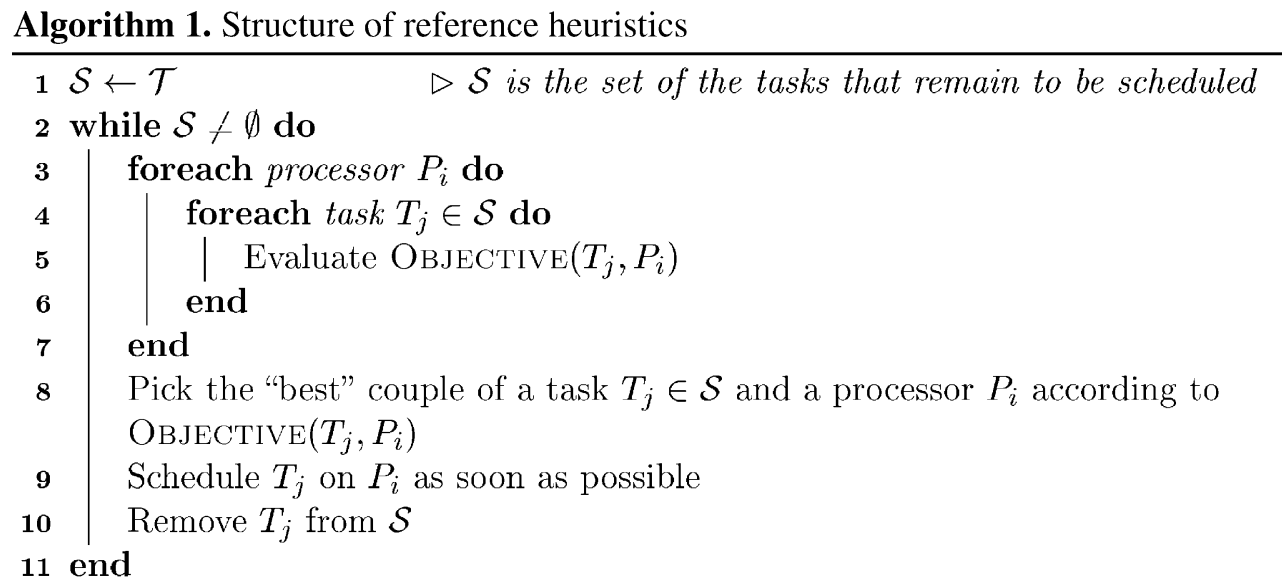

\subsubsection{Objective function}

For all the heuristics, the objective function is the same. Овлестіve $\left(T_{j}, P_{i}\right)$ is indeed the minimum completion time (MCT) of task $T_{j}$ if mapped on processor $P_{i}$. Of course, the computation of this completion time takes into account:

(1) the files required by $T_{j}$ that are already available on $P_{i}$ (we assume that any file that once was sent to processor $P_{i}$ is still available and do not need to be resent);

(2) the time needed by the master to send the other files to $P_{i}$, knowing what communications are already scheduled;

(3) the tasks already scheduled on $P_{i}$.

\subsubsection{Chosen task}

The heuristics only differ by the definition of the "best" couple $\left(T_{j}, P_{i}\right)$. More precisely, they only differ by the definition of the "best" task. Indeed, the "best" task $T_{j}$ is always mapped on its most favorable processor (denoted $P\left(T_{j}\right)$ ), i.e., on the processor which minimizes the objective function:

$$
\operatorname{ObJective}\left(T_{j}, P\left(T_{j}\right)\right)=\min _{1 \leqslant q \leqslant p} \operatorname{OBJective}\left(T_{j}, P_{q}\right)
$$

Here is the criterion used for each reference heuristic:

Min-min. The "best" task $T_{j}$ is the one minimizing the objective function when mapped on its most favorable processor; shortest tasks are scheduled first to avoid gaps at the beginning of the schedule:

$\operatorname{ObJective~}\left(T_{j}, P\left(T_{j}\right)\right)=\min _{T_{k} \in \mathscr{Y}} \min _{1 \leqslant l \leqslant p} \operatorname{OBJective}\left(T_{k}, P_{l}\right)$

Max-min. The "best" task is the one whose objective function, on its most favorable processor, is the largest; the idea is that a long task scheduled at the end would delay the end of the whole execution:

$\operatorname{ObJective}\left(T_{j}, P\left(T_{j}\right)\right)=\max _{T_{k} \in \mathscr{Y}} \min _{1 \leqslant l \leqslant p} \operatorname{OBJeCtive}\left(T_{k}, P_{l}\right)$

Sufferage. The "best" task is the one which will be the most penalized if not mapped on its most favorable processor but on its second most favorable processor, i.e., the "best" task is the one maximizing:

$\min _{P_{q} \neq P\left(T_{j}\right)}$ OвJеCtive $\left(T_{j}, P_{q}\right)-$ OBjective $\left(T_{j}, P\left(T_{j}\right)\right)$

with

$\operatorname{Objective}\left(T_{j}, P\left(T_{j}\right)\right)=\min _{1 \leqslant l \leqslant p} \operatorname{OBJective}\left(T_{j}, P_{l}\right)$

Sufferage II and Sufferage $X$. these are refined version of the Sufferage heuristic. The penalty of a task is no more computed using the second most 
favorable processor but by considering the first processor inducing a significant increase in the completion time. See $[1,2]$ for details.

\subsubsection{Computational complexity}

The loop on Step 3 of the reference heuristics computes the objective function for any pair of processor and task. For each processor, this computation has a worst case complexity of $O(|\mathscr{S}|+$ $|\mathscr{E}|)$, where $\mathscr{E}$ is the set of the edges representing the relations between files and tasks (see Section 2.1). Hence, the overall complexity of the heuristics is: $O(p \cdot n \cdot(n+|\mathscr{E}|))$. The complexity is even worse for Sufferage II and Sufferage $X$, as the processors must be sorted for each task, leading to a complexity of $O(p \cdot n \cdot(n \cdot \log p+|\mathscr{E}|))$.

\subsection{Structure of the new heuristics}

When designing new heuristics, we took special care to decreasing the computational complexity. The reference heuristics are very expensive for large problems. We aimed at designing heuristics which are an order of magnitude faster, while trying to preserve the quality of the schedules produced.
In order to avoid the loop on all the pairs of processors and tasks of Step 3 of the reference heuristics, we need to be able to pick (more or less) in constant time the next task to be scheduled. Thus we decided to sort the tasks a priori according to an objective function. However, since our platform is heterogeneous, the task characteristics may vary from one processor to the other. For example, Johnson's [9] criterion which splits the tasks into two sets (communication time smaller than, or greater than, computation time) depends on the processors characteristics. Therefore, we compute one sorted list of tasks for each processor. Note that this sorted list is computed a priori and is not modified during the execution of the heuristic.

Once the sorted lists are computed, we still have to map the tasks to the processors and to schedule them. The tasks are scheduled one-at-a-time. When we want to schedule a new task, on each processor $P_{i}$ we evaluate the completion time of the first task (according to the sorted list) which has not yet been scheduled. Then we pick the pair task/processor with the lowest completion time. This way, we obtain the structure of heuristics presented by Algorithm 2.

Algorithm 2. Structure of the new heuristics

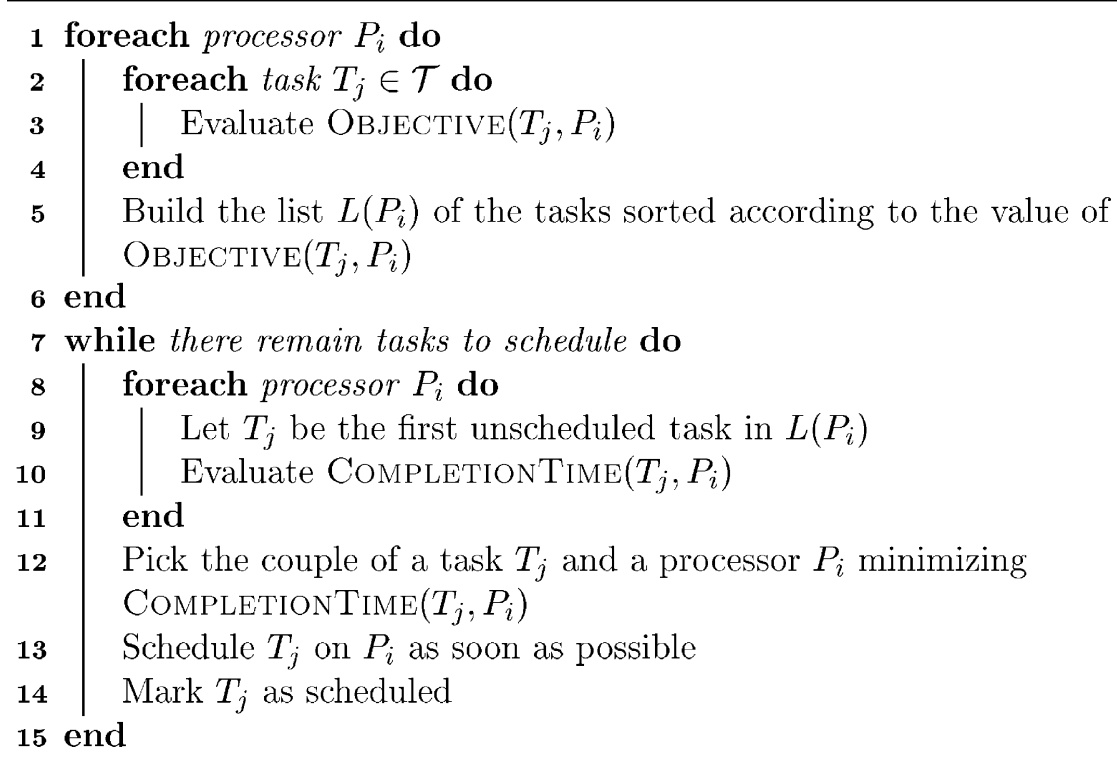


We still have to define the objective functions used to sort the tasks. This is the object of the next section.

\subsection{The objective functions}

The intuition behind the following six objective functions is quite obvious:

Duration. We just consider the overall execution time of the task as if it was the only task to be scheduled on the platform:

$\operatorname{OBJective}\left(T_{j}, P_{i}\right)=t_{j} \cdot w_{i}+\sum_{e_{k, j} \in \mathscr{E}} f_{k} \cdot c_{i}$.

The tasks are sorted by increasing objectives, which mimics the Min-min heuristic.

Payoff. When mapping a task, the time spent by the master to send the required files is payed by all the (waiting) processors as the master can only send files to a single slave at a time, but the whole system gains the completion of the task. Hence, the following objective function encodes the payoff of scheduling the task $T_{j}$ on the processor $P_{i}$ :

$\operatorname{ObJective}\left(T_{j}, P_{i}\right)=\frac{t_{j}}{\sum_{e_{k, j} \in \mathscr{E}} f_{k}}$.

The tasks are sorted by decreasing payoffs. Note that the actual objective function to compute the payoff of scheduling task $T_{j}$ on processor $P_{i}$ would be: $\operatorname{OBJective~}\left(T_{j}, P_{i}\right)=t_{j} \cdot w_{i} /\left(\sum_{e_{k, j} \in \delta} f_{k} \cdot c_{i}\right)$; as the factors $w_{i}$ and $c_{i}$ do not change the relative order of the tasks on a given processor, we just dropped these factors. Furthermore, the order of the tasks does not depend on the processor, so only one sorted list is required with this objective function.

Advance. To keep a processor busy, we need to send it all the files required by the next task that it will process, before it ends the execution of the current task. Hence the execution of the current task must be larger than the time required to send the files. We tried to encode this requirement by considering the difference of the computationand communication-time of a task, i.e., the advance earned due to the execution of this task. Hence the objective function:

$\operatorname{ObJective}\left(T_{j}, P_{i}\right)=t_{j} \cdot w_{i}-\sum_{e_{k, j} \in \mathscr{E}} f_{k} \cdot c_{i}$.
The tasks are sorted by decreasing objectives.

Johnson. We sort the tasks on each processor as Johnson does for a two-machine flow shop (see Section 3.1).

Communication. As the communications may be a bottleneck we consider the overall time needed to send the files a task depends upon as if it was the only task to be scheduled on the platform:

$\operatorname{ObJective}\left(T_{j}, P_{i}\right)=\sum_{e_{k, j} \in \mathscr{E}} f_{k}$.

The tasks are sorted by increasing objectives, like for Duration. As for Payoff, the sorted list is processor independent, and only one sorted list is required with this objective function. This simple objective function is inspired by the work in [8] on the scheduling of homogeneous tasks on an heterogeneous platform.

Computation. Symmetrically, we consider the execution time of a task as if it was not depending on any file:

Objective $\left(T_{j}, P_{i}\right)=t_{j}$.

The tasks are sorted by increasing objectives. Once again, the sorted list is processor independent.

\subsection{Additional policies}

In the definition of the previous objective functions, we did not take into account the fact that the files are potentially shared between the tasks. Some of them will probably be already available on the processor where the task is to be scheduled, at the time-step we would try to schedule it. Therefore, on top of the previous objective functions, we add the following additional policies. The goal is (to try) to take file sharing into account.

Shared. When a file is sent to a processor, it is beneficial to all tasks depending upon it. We try to express this idea by using, in the objective functions, weighted sizes for the files. The weighted size of a file is obtained by dividing its size by the number of tasks that are dependent upon the file. For example, the objective function for Duration + shared is

$t_{j} \cdot w_{i}+\sum_{e_{k, j} \in \mathscr{E}} \frac{f_{k}}{\left|\left\{T_{l} \mid e_{k, l} \in \mathscr{E}\right\}\right|} \cdot c_{i}$. 
Readiness. For a given processor $P_{i}$, and at a given time, the "ready" tasks are the ones whose files are already all on $P_{i}$. Under the Readiness policy, if there is any ready task on processor $P_{i}$ at Step 9 of the heuristics, we pick one ready task instead of the first unscheduled task in the sorted list $L\left(P_{i}\right)$.

Locality. In order to try to decrease the amount of file replication, we (try to) avoid mapping a task $T_{j}$ on a processor $P_{i}$ if some of the files that $T_{j}$ depends upon are already present on another processor. To implement this policy, we modify Step 9 of the heuristics. Indeed, we no longer consider the first unscheduled task in $L\left(P_{i}\right)$, but the next unscheduled task which does not depend on files present on another processor. If we have scanned the whole list, and if there remains some unscheduled tasks, we restart from the beginning of the list with the original task selection scheme (first unscheduled task in $L\left(P_{i}\right)$ ).

Finally, we obtain as many as 44 variants, since any combination of the three additional policies may be used for the six base objective functions, except for Shared which does not impact Computation.

\subsection{Computational complexity}

Overall, there are $|\mathscr{E}|$ dependence relations between tasks and files. Thus, computing the value of an objective function for all tasks on all processors has a cost of $O(p \cdot(n+|\mathscr{E}|))$, except for heuristic Computation for which the cost is $\mathrm{O}(p \cdot n)$, as the relations between tasks and files are not considered. So the construction of all the sorted lists has a cost of $O(p \cdot n \cdot \log n+p \cdot|\mathscr{E}|)$ for heuristics Duration, Advance, and Johnson ( $p$ lists), of $O(n \cdot \log n+|\mathscr{E}|)$ for heuristics Payoff and Communication (a single list), and of $O(n \cdot \log n)$ for heuristic Computation (a single list). If we denote by $\Delta T$, one plus the maximum number of files that a task depends upon, the execution of the loop at Step 7 of the heuristics (see Algorithm 2) has an overall cost of $O(p \cdot n \cdot \Delta T)$. Note that $n \cdot \Delta T \geqslant|\mathscr{E}|$. Hence the overall execution time of the heuristics is:

$O(p \cdot n \cdot(\log n+\Delta T))$ for heuristics using several lists (Duration, Advance, and Johnson), and

$O(n \cdot \log n+p \cdot n \cdot \Delta T)$

for the others (Payoff, Communication, and Computation). We have replaced the term $n+|\mathscr{E}|$ in the complexity of the reference heuristics by the term $\log n+\Delta T$. The experimental results will assert the gain in complexity.

Note that all the additional policies can be implemented without increasing the complexity of the base cases. It is obvious for Shared. Readiness can be implemented without overhead if we maintain, for all tasks on all processors, a counter of the number of files that are missing for the task on the processor. Each time a file is sent, this counter is updated. When a counter comes to zero, the corresponding task is moved into the set of tasks that are ready for the processor. For Locality, one just have to remember, for each task, where the files it depends upon have already been sent: on a single processor (we keep its number), or already spread over several processors. When a file is sent to some slave, this flag is updated for all tasks depending upon the file. In each case, for all tasks on a given processor, there are at most $|\mathscr{E}|$ updates. The additional cost of the Readiness and Shared policies is thus of $O(p \cdot|\mathscr{E}|)$, which is completely absorbed by the overall complexity of the heuristics.

\section{Experimental results}

In order to compare our heuristics and the reference heuristics, we have simulated their executions on randomly built platforms and graphs. We have conducted a very large number of experiments, which we summarize in this section.

\subsection{Experimental platforms}

Processors. We have recorded the cycle time of the different computers used in our laboratories (in Lyon and Strasbourg). From this set of values, we randomly pick values whose difference with the mean value was less than the standard deviation. 
This way we define a realistic and heterogeneous set of 20 processors.

Communication links. The 20 communication links between the master and the slave are built along the same principles as the set of processors.

Communication to computation cost ratio. The absolute values of the communication link bandwidths or of the processors speeds have no meaning (in real life they are application dependent and must be pondered by application characteristics). We are only interested by the relative values of the processors speeds, and of the communication links bandwidths. Therefore, we normalize processor and communication average characteristics. Also, we arbitrarily impose the communicationto-computation cost ratio, so as to model three main types of problems: computation intensive $($ ratio $=0.1)$, communication intensive (ratio $=$ $10)$, and intermediate (ratio $=1$ ).

\subsection{Application graphs}

We run the heuristics on the following four types of application graphs. In each case, the sizes of the files and tasks are randomly and uniformly taken between 0.5 and 5 .

The graphs are schematically represented in Fig. 7.

Forks. Each graph contains 100 fork graphs, where each fork graph is made up of 20 tasks depending on a single and same file (Fig. 7(a)).

Two-one. Each task depends on exactly two files: one file which is shared with some other tasks, and one unshared file (Fig. 7(b)).

Partitioned. The graph is divided into 20 chunks of 100 tasks, and in each chunk each task ran- domly depends on 1 up to 10 files. The whole graph contains at least 20 different connected components (Fig. 7(c)).

Random. Each task randomly depends on 1 up to 50 files (Fig. 7(d)).

Our objective is to use graphs representative of a large application class. The fork graphs represent embarrassingly parallel applications. The two-one graphs come from the original papers by Casanova et al. $[2,1]$. The partitioned graphs deal with applications encompassing some regularity. The random graphs are for totally irregular applications. Each of our graphs contains 2000 tasks and 2500 files, except for the fork graphs which also contain 2000 tasks but only 100 files.

In order to avoid any interference between the graph characteristics and the communication-tocomputation cost ratio, we normalize the sets of tasks and files so that the sum of the file sizes equals the sum of the task sizes times the communication-to-computation cost ratio.

\subsection{Results}

Table 1 summarizes all the experiments. In this table, we report the performance of the best ten heuristics, together with their cost (i.e., their CPU time). This is a summary of 12,000 random tests (1000 tests over all four graph types and three communication-to-computation cost ratios). Each test involves 49 heuristics (5 reference heuristics and 44 combinations for our new heuristics). For each test, we compute the ratio of the performance of all heuristics over the best heuristic, which gives us a relative performance. The best heuristic differs from test to test, which explains why no heuristic
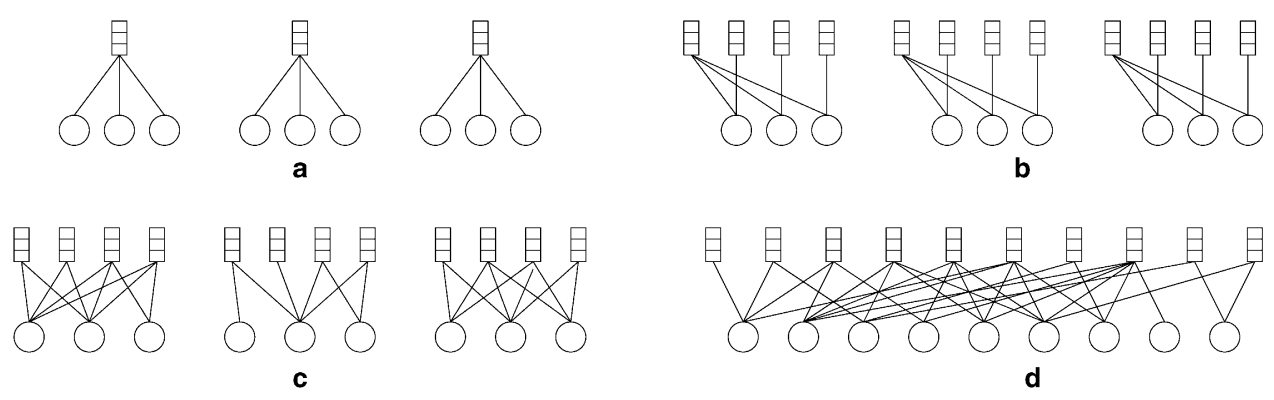

Fig. 7. The four type of application graphs used in the simulations: (a) forks (b) two-one (c) partitioned and (d) random. 
Table 1

Relative performance and cost of the best 10 heuristics

\begin{tabular}{|c|c|c|c|c|}
\hline Heuristic & Relative performance & Standard deviation & Relative cost & Standard deviation \\
\hline Sufferage & 1.110 & 0.1641 & 376.7 & 153.4 \\
\hline Min-min & 1.130 & 0.1981 & 419.2 & 191.7 \\
\hline Computation + readiness & 1.133 & 0.1097 & 1.569 & 0.4249 \\
\hline Duration + locality + readiness & 1.133 & 0.1295 & 1.499 & 0.4543 \\
\hline Duration + readiness & 1.133 & 0.1299 & 1.446 & 0.3672 \\
\hline Payoff + shared + readiness & 1.138 & 0.1260 & 1.496 & 0.6052 \\
\hline Payoff + readiness & 1.139 & 0.1266 & 1.246 & 0.2494 \\
\hline Payoff + shared + locality + readiness & 1.145 & 0.1265 & 1.567 & 0.5765 \\
\hline Payoff + locality + readiness & 1.145 & 0.1270 & 1.318 & 0.2329 \\
\hline Computation + locality + readiness & 1.147 & 0.1234 & 1.618 & 0.4749 \\
\hline
\end{tabular}

in Table 1 can achieve an average relative performance exactly equal to 1 . In other words, the best heuristic is not always the best of each test, but it is closest to the best of each test on average. The optimal relative performance of 1 would be achieved by picking, for any of the 12,000 tests, the best heuristic for this particular case. (For each test, the relative cost is computed along the same guidelines, using the fastest heuristic.) Note that we report extensive simulation results in [12].

We see that Sufferage gives the best results: on average, it is within $11 \%$ of the relative optimal of 1. The next nine heuristics closely follow: they are within $13-14.7 \%$ of the relative optimal. Out of these nine heuristics, only Min-min is a reference heuristic. Max-min is almost the worst heuristic. This can be explained as follows: in the beginning, this heuristic advantages tasks that have no files on any slave, generating lots of communications, and thereby delaying the execution of the following tasks. Despite the additional time spent to compute the schedule, Sufferage II and Sufferage $X$ do not achieve as good performance as Sufferage. The variants of Sufferage base their scheduling choices on a prediction of what would happen if these choices were not selected. This prediction does not take into account the possible file transfers due to the scheduling of other tasks. Thus it is not surprising that Sufferage II and Sufferage X, which try to make predictions in a longer term, make bigger mistakes, and finally achieve worst results.

Concerning our new heuristics, we can see that the performance of those appearing in Table 1 closely follows the performance of Min-min. Furthermore, the standard deviations are lower for our heuristics, which reflects a greater stability of the results.

On the average, Duration, Computation, and Payoff (along with Readiness) achieve the best performances. Communication lags well behind. However, the results for Computation and Communication must be nuanced. The performance of Computation degrades as the communicationto-computation cost ratio increases, while it is the inverse for Communication. This is not surprising, when looking at the definition of these heuristics. Computation only uses the computation time of the tasks, hence is more adapted to problems that are computation intensive. A similar explanation holds for Communication. Duration, which combines both approaches, is more stable across the different communication-to computation cost ratios.

Advance, Payoff, and Johnson are all based on the same observation: one should try to maximize the overlap of the communications by the computations. They, however, achieve performances that are very different. In any case, Advance and its variants perform badly in comparison with the other heuristics. Among those three heuristics, Payoff is the one with the best performance. It seems that Advance tends to favor tasks with large communication times. This observation is verified by the fact that it is improved by the Shared policy, which reduces the importance of communication times. On the contrary, Payoff may also favor a task with a large communication time, but only if the advance brought by its computation is really important (much more than what is required by Advance to schedule this task). Regarding Johnson, it achieves intermediate performance between Pay- 
off and Advance. Its best variant (Readiness) achieves an average relative performance of 1.172. Johnson's algorithm [9], which is optimal without file sharing, does not adapt well to the general case, where tasks may share files.

A close observation of the results shows us that the differences between the heuristics are more significant when the communication-to-computation cost ratio is low. In the opposite case, it is likely that the communications from the master become the true bottleneck of all scheduling strategies.

As for the additional policies, Readiness brings a real gain. In comparison with the base heuristics, this policy enhances the performances by more than $8 \%$ on average, except for Communication for which the gain is negligible. Readiness, whose objective is to reduce the number of replicated files, reveals itself as specially effective. We can observe that, with Communication, tasks roughly depending on a same set of files tend to be put together in the task lists. So, when such a task is scheduled on some slave, it induces the transfer of the concerned files, and the other tasks which follow wind up at the beginning of the list. Readiness has thus little influence. This phenomenon is perfectly illustrated for the graphs of type Forks. For those graphs of type Forks, the difference between Communication and Computation is the most evident. Both heuristics perform badly in their basic versions, but Computation + readiness outperforms by $20 \%$ all the other heuristics, including the reference heuristics. It is because of these results for the Fork graphs that Computation + readiness ended up in Table 1, whereas Communication + readiness did not.

Locality does practically not change the performances of the heuristics. It seems that this policy, whose goal was to improve the locality with respect to the use of the files, is not aggressive enough. Shared, our last variant, does only affect significantly Advance, Duration, and Johnson: the former is improved (but does not become of good quality), while the last ones are degraded. With Duration and Johnson, it is likely that Shared advantages tasks with large communication times, instead of those depending on highly shared files, as we wanted to. Another approach to better take file sharing into account, would be to reevaluate the ordering of the tasks on the processors, as files are being transferred.

In Table 1, we also report the computational costs of the heuristics (CPU time needed by each heuristic). The theoretical analysis is confirmed: our new heuristics are at least an order of magnitude faster than the reference heuristics.

As a conclusion, given their good performance compared to Sufferage, we believe that the eight new variants listed in Table 1 provide a very good alternative to the costly reference heuristics. The Readiness policy brings a large gain. As for the base heuristics, the simplest idea (among those that we evaluated) seems to work best: heuristics that only use an estimation of the execution time of the tasks on the processors. Depending upon the application graph, Computation + readiness and Duration + readiness are the recommended heuristics. The former performs better on Fork graphs, but the latter gives more stable results for all types of graphs.

\section{Conclusion}

In this paper, we have dealt with the problem of scheduling a large collection of independent tasks, that may share input files, onto heterogeneous clusters. On the theoretical side, we have shown new complexity results. On the practical side, we have improved upon the heuristics proposed by Casanova et al. $[1,2]$. We have succeeded in designing a collection of new heuristics which have similar performances but whose computational costs are an order of magnitude lower.

This work, as the one of Casanova et al., was limited to the master-slave paradigm. It is intended as a first step towards addressing the challenging situation where

- input files are distributed among several file servers (several masters) rather than being located on a single master,

- communication can take place between computational resources (slaves) in addition to the messages sent by the master(s): some slave may well propagate files to another slave while computing. 
We hope that the ideas introduced when designing our heuristics will prove useful for this difficult scheduling problem. As shown by the preliminary results reported in [13], much work remains to be done to design efficient mapping and scheduling strategies in a fully decentralized environment.

\section{References}

[1] H. Casanova, A. Legrand, D. Zagorodnov, F. Berman, Heuristics for scheduling parameter sweep applications in Grid environments, in: Ninth Heterogeneous Computing Workshop, IEEE Computer Society Press, Silver Spring, MD, 2000, pp. 349-363.

[2] H. Casanova, A. Legrand, D. Zagorodnov, F. Berman, Using simulation to evaluate scheduling heuristics for a class of applications in grid environments, Research Report RR-1999-46, LIP, ENS Lyon, France, 1999.

[3] F. Berman, High-performance schedulers, in: I. Foster, C. Kesselman (Eds.), The Grid: Blueprint for a New Computing Infrastructure, Morgan-Kaufmann, Los Altos, CA, 1999, pp. 279-309.

[4] M. Maheswaran, S. Ali, H.J. Siegel, D. Hensgen, R.F. Freund, Dynamic matching and scheduling of a class of independent tasks onto heterogeneous computing systems, in: Proceedings of the Eight Heterogeneous Computing Workshop, IEEE Computer Society Press, Silver Spring, MD, 1999, pp. 30-44.

[5] T.D. Braun, H.J. Siegel, N. Beck, L.L. Bölöni, M. Maheswaran, A.I. Reuther, J.P. Robertson, M.D. Theys, B. Yao, D. Hensgen, R.F. Freund, A comparison of eleven static heuristics for mapping a class of independent tasks onto heterogeneous distributed computing systems, Journal of Parallel and Distributed Computing 61 (6) (2001) 810-837.

[6] B.A. Shirazi, A.R. Hurson, K.M. Kavi (Eds.), Scheduling and Load Balancing in Parallel and Distributed Systems, IEEE Computer Society Press, Silver Spring, MD, 1995.

[7] Ph. Chrétienne, E.G. CoffmanJr., J.K. Lenstra, Z. Liu (Eds.), Scheduling Theory and its Applications, John Wiley and Sons, New York, 1995.

[8] O. Beaumont, A. Legrand, Y. Robert, A polynomial-time algorithm for allocating independent tasks on heterogeneous fork-graphs, in: ISCIS XVII, Seventeenth International Symposium on Computer and Information Sciences, CRC Press, Boca Raton, 2002, pp. 115-119.

[9] S.M. Johnson, Optimal two- and three-stage production schedules with setup times included, Naval Research Logistics Quarterly 1 (1954) 61-68.

[10] E.M. Macambira, C.C. de Souza, The edge-weighted clique problem: valid inequalities, facets and polyhedral computations, European Journal of Operational Research 123 (2) (2000) 346-371.
[11] M.R. Garey, D.S. Johnson, Computers and Intractability, a Guide to the Theory of NP-Completeness, W.H. Freeman and Company, New York, 1979.

[12] A. Giersch, Y. Robert, F. Vivien, Scheduling tasks sharing files on heterogeneous clusters, Research Report RR-200328, LIP, ENS Lyon, France, also available as INRIA Research Report 4819, 2003.

[13] A. Giersch, Y. Robert, F. Vivien, Scheduling tasks sharing files from distributed repositories, in: Euro-Par'04: Parallel ProcessingLecture Notes in Computer Science, Springer Verlag, Berlin, 2004, pp. 148-159.

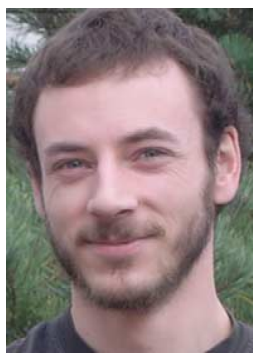

Arnaud Giersch was born in 1977 in Colmar, France. He obtained a $\mathrm{PhD}$ thesis from Louis Pasteur University of Strasbourg in December 2004. He is currently a postdoctoral researcher in the Computer Science Laboratory ICPS/LSIIT at Louis Pasteur University of Strasbourg. His main research interests are scheduling and load balancing techniques for heterogeneous platforms.

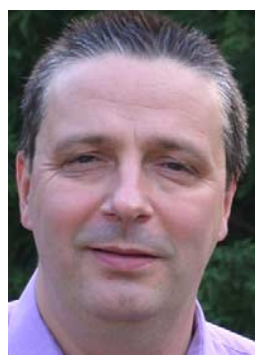

Yves Robert was born in 1958 in Lyon, France. He obtained a $\mathrm{PhD}$ thesis from Institut National Polytechnique de Grenoble in January 1986. He is currently a full professor in the Computer Science Laboratory LIP at ENS Lyon. $\mathrm{He}$ is the author of four books, 90 papers published in international journals, and 115 papers published in international conferences. His main research interests are scheduling techniques and parallel algorithms for clusters and grids. He is a senior member of IEEE, and serves as an associate editor of IEEE TPDS.

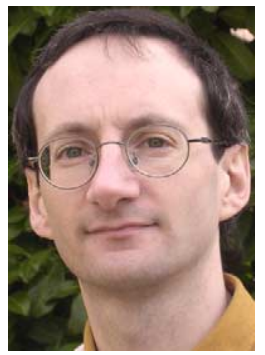

Frédéric Vivien was born in 1971 in Saint-Brieuc, France. He obtained a $\mathrm{PhD}$ thesis from École normale supérieure de Lyon in 1997. From 1998 to 2002 , he had been an associate professor at Louis Pasteur University of Strasbourg. He spent the year 2000 working in the Computer Architecture Group of the MIT Laboratory for Computer Science. He is currently a full reseacher at INRIA. His main research interests are scheduling techniques, parallel algorithms for clusters and grids, and automatic compilation/parallelization techniques. 